\title{
Information Standards to support Application and Enterprise Interoperability for the Smart Grid
}

\author{
Nigel Hargreaves, Student Member, IEEE; Gareth Taylor, Member, IEEE; Alex Carter.
}

\begin{abstract}
Current changes in the European electricity industry are driven by regulatory directives to reduce greenhouse gas emissions, at the same time as replacing aged infrastructure and maintaining energy security. There is a wide acceptance of the requirement for smarter grids to support such changes and accommodate variable injections from renewable energy sources. However the design templates are still emerging to manage the level of information required to meet challenges such as balancing, planning and market dynamics under this new paradigm. While secure and scalable cloud computing architectures may contribute to supporting the informatics challenges of the smart grid, this paper focuses on the essential need for business alignment with standardised information models such as the IEC Common Information Model (CIM), to leverage data value and control system interoperability. In this paper we present details of use cases being considered by National Grid, the GB transmission system operator for information interoperability in pan-network system management and planning.
\end{abstract}

Index Terms - Common Information Model, Interoperability, Power system management, Smart grid.

\section{INTRODUCTION}

The challenges faced in renewing the electricity industry broadly fall into two categories: the task of rewiring electricity networks to accommodate new, decentralised patterns of generation, and the need for open electricity markets which support more consumer interaction and the sale of electricity across interconnected networks [1][2]. There is general agreement that the unprecedented combination of challenges to secure electricity supplies in future has given rise to the need for smarter electricity networks carrying transmission level to domestic supply voltages. But the term smart grid has no universally accepted definition although it imparts the need for inter-connectivity through intelligent use of network and system interoperability. The UK Electricity Networks Strategy Group (ENSG), have identified that a smart grid should facilitate wider control over energy management by providing

N. B. Hargreaves is with Brunel University, London, and gratefully acknowledges the financial support of his sponsor National Grid, UK. (email: nigel.hargreaves@brunel.ac.uk)

G. A. Taylor is with Brunel University, London (email: gareth.taylor@brunel.ac.uk)

A. Carter is with National Grid, Electricity Control Centre, UK (email: alex.carter@uk.ngrid.com) tariff-based choices for consumers and feature a holistic communications system providing greater clarity of the grid state to allow it to operate in a way coherent with decarbonisation priorities [3]. A major challenge to the smart grid is the need to interoperate a variety of electricity and information networks that will have to support business needs from a variety of stakeholders while being flexible enough to meet the greater demands from the widening scope of electrification in the future. From this standpoint, serving the critical interaction between business needs and the physical reality of very large, interconnected electricity networks pulls focus on the information exchanges to enable such 'end-to-end interoperability'. There are several ways to represent the cyber-physical nature of a smart grid by breaking it down into reference frameworks. This is a fundamental step in guiding the transformation of existing electricity networks into smarter ones and reflects the direction taken by organisations such as the Joint Working Group (JWG) for smart grids in the European Union [4] and the National Institute of Standards and Technology (NIST) in the USA [5]: both acknowledge the large number (75-100) of existing industry standards requiring integration to support the desired level of interoperability across the spectrum of smart grid elements - from hardware components to information exchange to business processes.

Following the format of the smart grid interoperability framework, as laid out by the GridWise Architecture Council (GWAC) [6], we present within the wider framework of standards, the IEC Common Information Model (IEC 61970, 61968) and associated smart grid information standards (Fig.1.), which give direction to power utilities developing scalable information interoperability architectures internally and externally, to build the necessary capability required to operate effectively as part of a smart grid [7] [8].

This paper will report on examples of how National Grid's business objectives towards 2020, [9], are being addressed by the development of scalable and interoperable enterprise information management (EIM) processes to meet the challenges outlined above within the context of the GB smart grid. Use cases for these information processes include:

- The exchange of network model information between National Grid and the GB regional Distribution Network Operators (DNOs).

- The exchange of network model information between National Grid and Coreso, the W European Regional Coordination Service Centre [10]. 
Economic/Regulatory Policy

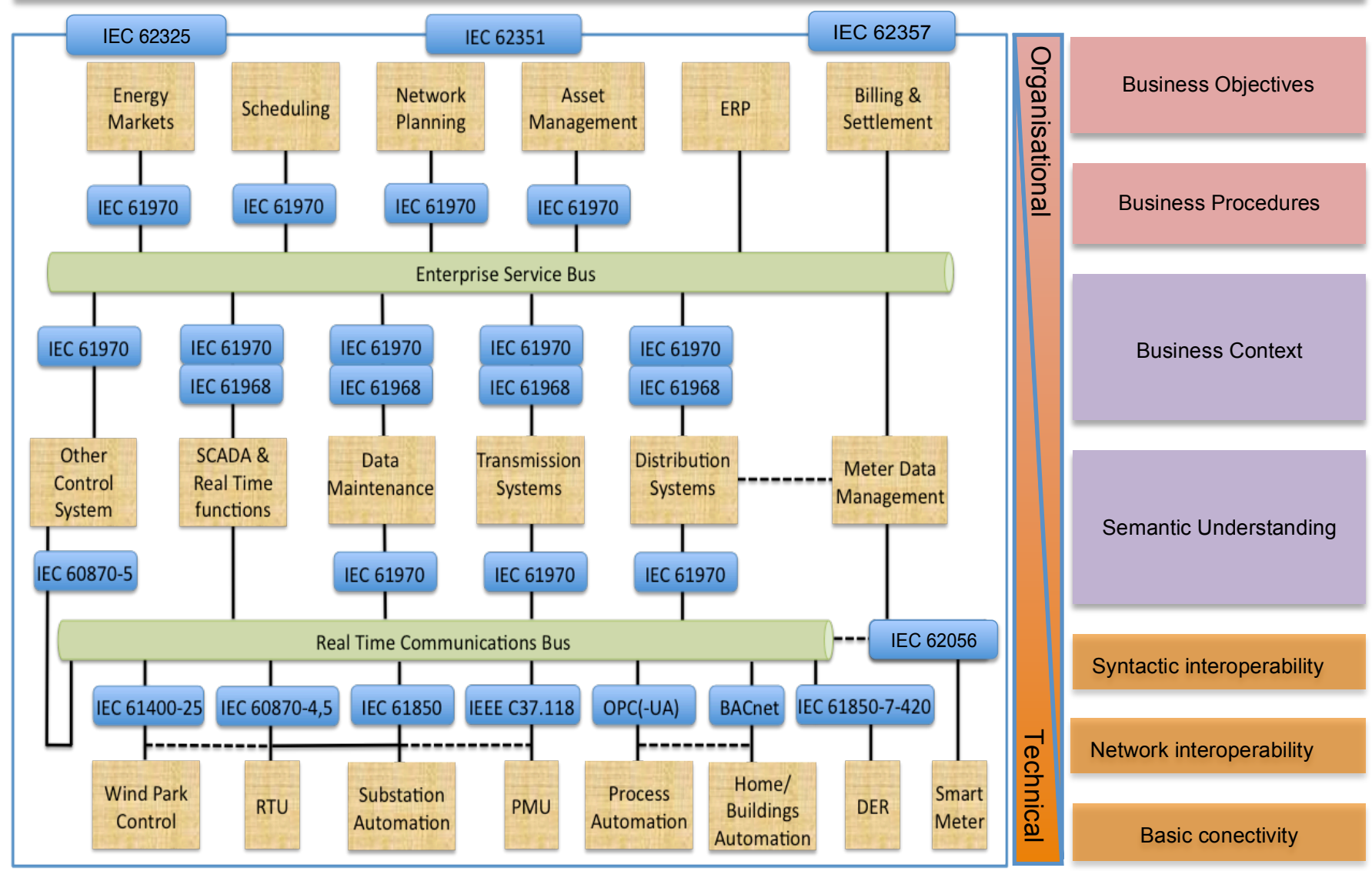

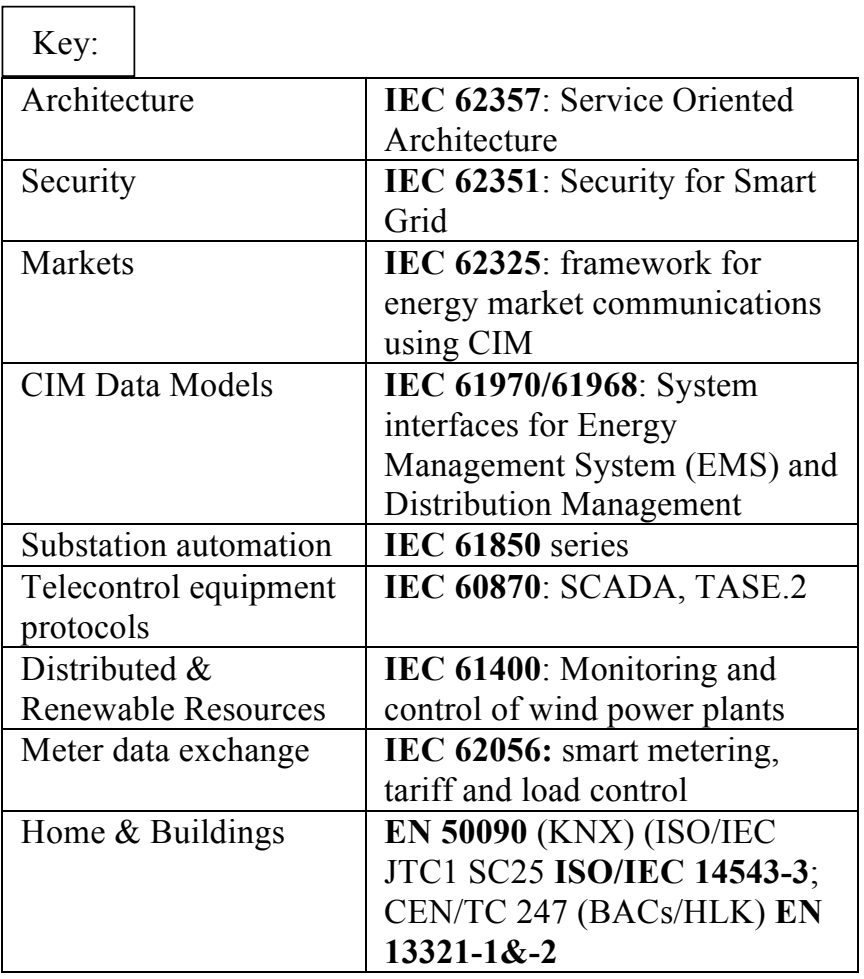

Fig. 1. A standards-driven interoperability architecture for smart grid Transmisson and Distribution Energy Management Systems. Adapted from: JWG smart grid report V1.0, 2011 [4], GridWise Architecture Council [6].

\section{NATIONAL GRID'S APPROACH TO SMART GRID INTEGRATION}

National Grid (NG) are developing a strategy calling for Enterprise Information Management (EIM) to operate critical national infrastructure within an era of radically changing demands on the GB electricity industry. Analysis of business objectives and the corresponding need for information exchange are required steps in a 'joined-up' process of standards implementation, offering the potential for creating an enterprise more integrated and responsive to the direction corporate, market and regulatory forces intend to drive it. Utilities which are able to respond to changes in regulatory and corporate business objectives most flexibly through endto-end interoperability, are also likely to succeed in the dynamic conditions making up the smart grid. National Grid aims to develop scalable information architecture for its part in the developing European smart grid.

Implementation of the CIM will only serve as part of the interoperability solution in transiting from a legacy of pointto-point system architectures, which have evolved through autonomous system replacement cycles [11]. The intention of enabling flexible access to network operational and planning information across the utility and its partners is perceived as essential to enabling NG's smart grid capability. A model 
driven approach to information management will reduce the amount complex legacy systems restrict access to data, often resulting in data-islanding and duplication.

The need for high-level managerial backing and supervision is called for in implementing a process which will challenge the prevalent company business model to operate differently, as greater coherence between Objectives, Capability, Functionality, Services, Architecture, Information and Hardware extends. Some of the initial business objectives for discussion in this paper are given below:

A. Offer trusted access to databases of shared network models for use by distribution network operator (DNO) transmission owner (TO) and other transmission system operator (TSO) clients.

B. Offer automated data model exchange with regional network coordination service centres such as Coreso.

C. Reduce separation between off-line planning and online control, network models.

\section{BUSINESS USE CASES FOR INITIAL MODEL DRIVEN DATA INTEGRATION AT NATIONAL GRID}

A. Under the GB Grid Code [12], which sets out operational procedures and principles directing NG's relationship with all users of the GB transmission system, there is a requirement for network configuration information to be submitted by the 14 regional DNOs on an annual basis. This information, which is often exchanged in a variety of file formats, is critical to update NG's visibility and planning of the demands likely to be placed on the transmission system for the year ahead. After integrating the DNO data into its own model, NG return network models reduced to the boundaries with the DNO networks, to refresh DNOs visibility of the GB transmission system at their own interfaces. Offshore Transmission System Owners (OFTOs), connecting large windfarms of over $100 \mathrm{MW}$ output capacity are treated similarly under the same governance agreement. These regulatory requirements, coupled with the transition of radial distribution networks to ones which are increasingly meshed (and liable to carry flows in both directions due to the presence of a growing amount of embedded generation), have given rise to the need for a greater visibility by NG of network dynamics at lower voltage levels.

Response: National Grid, in conjunction with Brunel University, Oxford eResearch Centre and Open Grid Systems have simulated a network model exchange with UK Power Networks through a trusted cloud infrastructure [13]. This project demonstrated that network models could be exchanged between utilities via a secure private cloud infrastructure built upon an open source solution - Eucalyptus. The Cimphony application from Open Grid Systems [14] was used in the cloud to transform an original native network model, exported in PSS/e, into CIM and then perform validation against the ENTSO-e edition-1 CIM profile (based on CIM release $14 \mathrm{v} 2$, 2009). Thus simulating interoperability between the planning tools used by the TSO and DNO.

B. Coreso is a Regional Coordination Service Centre, based in Brussels, whose objective is to improve the security of supply in Western Europe by supporting regional TSOs through modeling the impacts of their transnational flow forecasts. As the GB transmission system becomes increasingly interconnected with the transmission systems of neighbouring countries, the issue of supergrid network security will also become more prominent. With interconnections to France and the Netherlands, NG now need to be vigilant to the effects of electrical flows transiting amongst these countries and Belgium.

Response: National Grid are leading work to export as a CIM XML file, constrained to the CIM ENTSO-e profile (2009, Edition 1), a reduced portion of the GB network. Covering the area in which the HVDC interconnectors are located, it will be merged with the boundary portions of their TSO partners within Coreso (Rte, elia, $50 \mathrm{~Hz}$ and Terna), using the Rte-developed Convergence software application. This functionality, which should go live in 2012, in addition to realtime EMS state estimator data snapshots, will provide Coreso with the necessary data to monitor transnational network boundary security for up to day +2 forecasts. This response could be affected by the outcome of the (C) below, in terms of how the data is exported.

C. The desire to converge the data used by models for both online EMS and offline planning and outage systems, as well as link offline models to real time measurements from telecontrol systems, is not unique to NG. Several system operators are pursuing this aim by developing centralised data models used by systems sharing information translated through CIM translation interfaces. In this case the data model effectively mirrors the data it is associated to, which may physically be stored in non-centralised databases.

Response: National Grid are currently at an early stage of planning conceptual architectures to bring data modeled by the EMS and offline planning systems together for validation, visualisation, integration and analysis. Initially this data will be modeled on the Common Power System Model profile (CPSM, using IEC61970-452) and the ENTSO-e profile (2009, Edition 1), exported from the EMS and the Offline Transmission Analysis (OLTA) systems. However, beyond that, a less constrained mapping of their internal databases to CIM may be achieved to widen the availability of these data, and associate them, where appropriate, to the unmodeled timeseries measurements entering from SCADA and other telecontrol equipment.

\section{CHALLENGES AHEAD IN PURSUING MODEL DRIVEN DATA INTEGRATION (MDI)}

Realising objectives such as those outlined above face a number of information handling and architectural design challenges including:

- Semantic alignment of object nomenclature within system proprietary data models to a common enterprise semantic model. The CIM can be used as a base model for this purpose.

- The handling of globally unique data identities, (GUIs, mRIDs), especially when data is shared between systems in different utilities. This issue is affected by data exchanged across company boundaries and could require 
the implementation of a centralised master identity registry.

- Database design to manage canonical associations found in the CIM as well as to link logical model data to timeseries generated data.

- Management of data models corresponding to a centralised data repository mapping of network models of differing resolutions such as switch-branch, nodal and switch-component data models.

- Management of proprietary extensions to CIM profiles, especially at times of implementing updates to the CIM release employed in the utility.

- Architectural design to support the best balance of utility-wide data access (employing Service Oriented Architectures and virtualisation of applications) against requisite security measures.

These are generic issues commonly faced by utilities engaging in MDI to build the smart grid [15]. With the likelihood of rapidly changing business objectives, architectural templates made up of a modular layering of service oriented processes of increasing granularity, may be called for to provide the necessary business flexibility [16].

\section{CONCEPTUAL INFORMATION MANAGEMENT ARCHITECTURES}

It is this scenario, which makes consideration of cloud computing attractive to power utility data management in the smart grid [17]. Although the information exchange use cases differ across metering, distribution and transmission networks, they all face a common future managing larger amounts of data more frequently in an increasingly stochastic operating environment - partly due to the variability imparted by renewable energy injections and embedded demand reduction technologies on the networks. Responding to data exchange requirements may not be the only challenge as scalable data processing requirements could be required to maintain operational systems under such network dynamics [18] [19]. There are various configurations of cloud infrastructures which are available to support these requirements but the issue of attestation to trustworthiness will need to be weighed within the chosen architecture [20] leading away from the use of public clouds (such as Amazon EC2) to private and private community clouds [21].

National Grid's smart grid information use cases, outlined in $\mathrm{A}$ and $\mathrm{B}$ above, could be addressed through the use of a private community cloud infrastructure based on the research carried out so far in the myTrustedCloud project (Fig. 2).

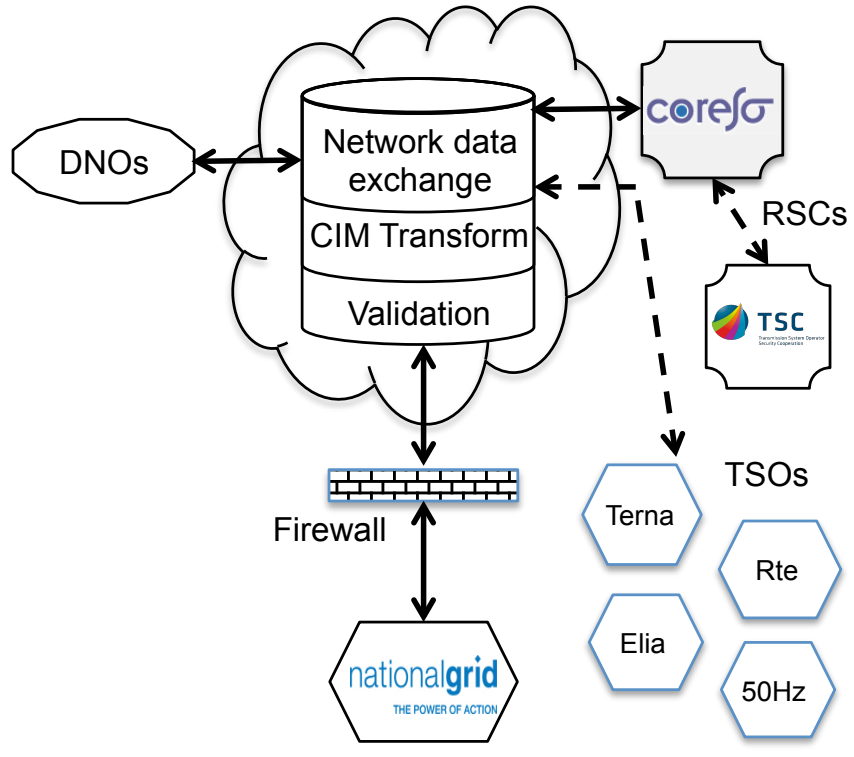

Fig. 2. Schematic of conceptual data exchanges between NG and GB DNOs, and Coreso. Dashed lines show the potential for project extension for data exchange to other European TSOs and TSC the regional service centre for central Europe.

\section{PROPOSED 10 STEP IMPLEMENTATION PLAN}

Business case $\mathrm{C}$ (above) may be considered as typical, but not the only justification for pursuing a top-down approach to EIM. National Grid as well as other system operators (SOs) have identified the desirability of having only 'one version of the truth' concerning data it uses to operate, plan, manage market activity and maintenance of its networks. The CIM offers an opportunity to realise this aim because of its detail and scope. Modeling data, which may otherwise have been duplicated on data islands within system silos is now possible. We propose an approach to such an ambitious objective, one which itself could change the business model and culture of operation, in a high-level 10-step implementation plan.

Step 1:

Define business smart grid objectives and derive known impacts on information management requirements; This will involve an assessment of pressures from market and regulatory forces within the business outlook time frame, as well as the need for systems to interoperate within usual technology refresh life-cycles. Analysis of the business processes and the information required for them to provide the necessary functional capability is implied.

Step 2:

Create EIM team; This team, comprising data modelers, engineers and information architects will be tasked with creating and enforcing use of the enterprise semantic model. They become the owners of the company information model, persisting the implementation of information interoperability standards through the lifecycles of the projects they oversee.

Step 3:

Identify the common horizontal business elements across multiple vertical projects; The scope of this exercise may take in transnational as well as national and internal projects which the business requires to interoperate. It is the groundwork for the implementation of a service oriented architecture later on. 
Step 4:

Identify and analyse into common and reusable elements key business processes (BPs); These are selected for offering the highest value in meeting the utility smart grid objectives identified in Step 1. This approach optimizes the effort involved in building the information model by starting with the areas of data integration which will bring most benefit to the business (see below).

Step 5:

Identify the information use cases required by the BPs above; This serves to give priority to the effort required next to implement modeling of the business process data using the standard information model.

Step 6:

Map data used in BP elements to CIM classes to build utility semantic data model; In this step the quality of data and metadata will be evident as it is mapped, offering an opportunity for data cleansing, validation and standardisation. Step 7:

Create company data architecture around the concept of a centralised information model repository; This step will be guided by the information use cases identified for the data in Step 4. It will address issues such as database design, storage technologies and information architectures required to meet the functional requirements called for by the business objectives.

Step 8:

Specify and develop interfaces and middleware, rationalized to a service oriented architecture; Completion of this step should result in the first stage of modeled data integration and offer opportunities measured against the values of BPs identified in Step 4.

Step 9:

Identify new information use cases from data now available in the central repository; The opportunity to extend the scope of information interoperability arises as new uses are made of existing data as well as widening the application of the developed architecture to include data from other systems.

Step 10:

Refresh the cycle of the standard information model implemented by moving to a later release; This requires a deepening commitment to model driven data integration by the utility and vendor partners as well as exercising version management of the CIM and ESM in use.

\section{FURTHER WORK}

The authors acknowledge that there is scope for further refinement of the implementation process for information standards within the utility and its external partners. Further work will therefore be derived from the practical implementation of standards such as IEC 61970 and 61968. There is also a need to capture and analyse the key business processes relating to the capability for the utility to achieve its smart grid objectives. This important stage of understanding the information flows which require modeling as a priority, demands further investigation.

\section{CONCLUSIONS}

In addressing the need for information management practices coherent with flexible business objectives required to operate successfully within the changing demands of the smart grid, we discuss the business case for model driven information integration within National Grid. While the requirement for data integration has been known for some years, we conclude the opportunity to achieve it has been impeded by the lack of an extensive and detailed set of information modeling standards upon which to base an enterprise semantic model. This principal requirement is now satisfied by the IEC CIM, and so the challenge of how to go about implementing it within a data integration strategy arises. We discuss the potential use of cloud and service oriented architectures, noting the requirement to balance trust and security measures within clouds proportionately with data and process virtualisation. Finally we propose a 10 step implementation plan for the CIM within a wider strategy of enterprise information management.

\section{REFERENCES}

[1] Institution of Engineering and Technology (IET), 2009. Smart Grid Thinking, Engineering \& Technology magazine pp 46-49. www.theiet.org/magazine

[2] A. Battaglini, J. Lilliestam, A. Haas, A. Patt, 2009. Development of SuperSmart Grids for a more efficient utilisation of electricity from renewable sources. Journal of Cleaner Production Volume 17, Issue 10, July 2009, Pages 911-918

[3] Electricity Networks Strategy Group (ensg), 2009. A Smart Grid Routemap.

[4] Joint Working Group, 2011. Final report of the JWG on Standards for Smart Grids. CEN/CENELEC/ETSI.

[5] National Institute of Standards and Technology (NIST), 2010 Framework and Roadmap for Smart Grid Interoperability Standards, Release 1.0. NIST Special Publication 1108, Office of the National Coordinator for Smart Grid Interoperability, USA.

[6] GridWise Interoperability Context-Setting Framework, 2008. GridWise Architecture Council, USA

[7] N. Hargreaves, G. Taylor, A. Carter, A. McMorran, 2011. Developing emerging standards for power system data exchangeto enable interoperable and scalable operational modelling and analysis. UPEC 2011, 5-9 September 2011, Soest, Germany.

[8] E. Haq, D. Haller, E. Rodriguez, 2010. Use of CIM Standard in Power Suystem Modeling \& Enterprise Wide Messaging at California ISO. Power and Engineering Society General Meeting, 2010 IEEE.

[9] National Grid, 2011. Consultation: Operating in 2020. http://www.nationalgrid.com/uk/Electricity/Operating+in+2020//Climate Change/Seealso/gonegreen2020.htm

[10] Coreso. http://www.coreso.eu/

[11] S. Neumann, T. Nielsen, 2010. CIM Interoperability Challenges. Power and Engineering Society General Meeting, 2010 IEEE.

[12] The GB Grid Code. http://www.nationalgrid.com/uk/Electricity/Codes/gridcode/gridcodedoc s/

[13] D. Wallom, M. Turilli, G. Taylor, N. Hargreaves, A. McMorran, A. Martin, A. Raun, 2011. myTrustedCloud: Trusted Cloud Infrastructure for Security-critical Computation and Data Management. $3^{\text {rd }}$ IEEE International Conference on Cloud Computing Technology and Science. 2011.

[14] Cimphony is a product of Open Grid Systems Ltd. Glasgow, UK.

[15] R. Khare, M. Khadem, S. Moorty, K, Methaprayoon, J, Zhu, 2011. Patterns and Practices for CIM Applications. Power and Engineering Society General Meeting, 2011 IEEE.

[16] L. Chang, T. Reynolds, B. Hong, 2010. Research on SOA-Based Integrated Strategy to enable the Smart Grid. International Conference on Cyber-Enabled Distributed Computing and Knowledge Discovery, 2010 . 
[17] S. Rusitschka, K. Eger, C. Gerdes, 2010. Smart Grid Data Cloud: A Model for Utilizing Cloud Computing in the Smart Grid Domain. Smart Grid Communications (SmartGridComm), IEEE, 2010.

[18] L. Zhang, X. Li, 2011. The Core of Constructing the Future Power Systems Computation Platform is Cloud Computing. International Conference on Mechatronic Science, Electric Engineering and Computer (MEC), China, 2011.

[19] M. Shah, R. Vaishnav, N. Rajagopal, K. Prasad, 2010. Delivering Power System Decision Support Tools Over the Web. Power and Energy Society General Meeting, IEEE, 2010.

[20] I. Abbadi, 2011. Operational Trust in Clouds' Environment. Symposium on Computers and Communications (ISCC), IEEE, 2011.

[21] L. Zheng, Y. Hu, C. Yang, 2011. Design and Research on Private Cloud Computing Architecture to Support Smart Grid. International Conference on Intelligent Human-Machine Systems and Cybernetics (IHMSC), 2011.

\section{IX.BIOGRAPHIES}

Nigel Hargreaves (Student M' 10) Obtained his MSc. In Climate Change Impacts and Sustainability from Brunel University, London in 2008 and his BSc. in Air Transport Engineering from the City University, London in 1981. He is currently studying for his $\mathrm{PhD}$. in Electrical Engineering while working within the Control Strategy Development team at National Grid. His area of interest is Enterprise Information Modeling and Management.

Gareth A. Taylor (M' 06) received his BSc degree from the University of London, UK in 1987 and MSc and PhD from the University of Greenwich, UK in 1992 and 1997, respectively. He was the National Grid UK post-doctoral scholar at Brunel University, UK from 2000-2003. He is currently a reader and course director within the Brunel Institute of Power Systems, Brunel University, UK. His research interests include power system and network optimization.

Alex Carter received his BEng and $\mathrm{PhD}$ degrees from the University of Bath, UK in 1992 and 1995 respectively. He joined National Grid UK in 1995 and is now looking at how National Grid needs to adapt its Great Britain System Operator role, processes and systems to the challenges of decarbonising the production of electricity. 